\title{
Potency of nano-antibacterial formulation from Sargassum binderi against selected human pathogenic bacteria
}

\author{
Sivakumar Sivagurunathan Moni ${ }^{1 *}$, Mohammad Firoz Alam², M M. Safhi², Aamena Jabeen', \\ Syeda Sanobar ${ }^{3}$, Rahimullah Siddiqui ${ }^{2}$, Remesh Moochikkal ${ }^{4}$
}

\begin{abstract}
${ }^{1}$ Department of Pharmaceutics, Faculty of Pharmacy, Jazan University, Jazan, Saudi Arabia, ${ }^{2}$ Department of Pharmacology and Toxicology, Faculty of Pharmacy, Jazan University, Jazan, Saudi Arabia, ${ }^{3}$ Department of Pharmacognosy, Faculty of Pharmacy, Jazan University, Jazan, Saudi Arabia, ${ }^{4}$ Department of Biology, Faculty of Science, Jazan University, Jazan,
\end{abstract}

Saudi Arabia

\begin{abstract}
Seaweeds constitutes an abundant marine reserve that can be harnessed as source of new pharmaceutical agents. Sargassum binderi Sonder ex J. Agardh is a brown seaweed that is predominantly available from December to March in the Red Sea, Jazan, Kingdom of Saudi Arabia (KSA). In this study, three extracts were isolated using three different techniques, and were subjected to antibacterial assay. The petroleum ether extract of Sargassum binderi was more effective against selected human pathogenic bacteria than the other extracts. Therefore, further studies were focused on developing oleic acid vesicles entrapped with the petroleum ether extract of Sargassum binderi, with the aim of enhancing its penetration property. Oleic acid vesicles were prepared by entrapping petroleum ether extract of Sargassum binderi using film hydration technique. The formulated vesicles were in nanoscale, and so were termed phyto-nanovesicles (PNVs). The spectrum of antibacterial activity of PNVs showed that it is a promising formulation against $S$. aureus, S. pyogenes, B. subtilis, E. coli, K. pneumoniae and $P$. aeruginosa. The microbial sensitivities to the PNVs was in the order E.coli $>$ B. subtilis $>S$. aureus $>S$. pyogenes $>$ K. pneumoniae $>P$. aeruginosa. Thus, the PNV formulation possesses promising and effective antimicrobial potential against human pathogenic bacteria.
\end{abstract}

Keywords: Red sea. Sargassum species. Fatty acid vesicles. Antibacterial formulations.

\section{INTRODUCTION}

Seaweeds are promising and potential sources of bioactive molecules and have been shown to have many pharmaceutical applications especially as antimicrobial agents (Namvar, Baharara, Mahdi, 2014; Maria, Elena, Herminia, 2016). Antibiotic resistance is a major problem worldwide, necessitating studies aimed at discovering new antibiotics/antibacterial agents (Sivakumar et al., 2016). However, complete eradication of anti-microbial resistance still remains a challenge. Many biochemical pathways have been used by bacteria to escape the lethal action of antibiotics through modification of the drug permeability across bacterial membranes (Cosgrove, 2006). In general, resistance factors in Gram-negative bacteria frequently

\footnotetext{
*Correspondence: S. S. Moni. Division of Immunology and Pharmaceutical Biotechnology, Department of Pharmaceutics, Faculty of Pharmacy, Jazan University, Jazan, Saudi Arabia. E-mail: drsmsivakumar@gmail.com
}

occur due to restricted permeability of the outer membrane to many antibacterial agents and antibiotics (Collis, Hall, 1995; Cosgrove, 2006). Studies suggest that the bacterial resistance against antibiotics/antibacterial agents is mostly due to Staphylococcus aureus, Streptococcus pyogenes, Mycobacterium tuberculosis, Escherichia coli, and Pseudomonas aeruginosa (Pelgrift, Friedman, 2013; Riley et al., 2012). Recent studies have shown that oleic acid vesicle is an excellent formulation for sustained delivery of entrapped drugs (Foziyah et al., 2010). Earlier-on, many reports demonstrated the importance of seaweeds in developing antibacterial agents.

Sargassum binderi Sonder ex J. Agardh belongs to the Sargassaceae family which is predominantly available in the sea of Jazan province, Jazan, KSA. In this study, a novel phyto-nanovesicle (PNV) formulation was prepared by entrapping crude phytoconstituents of Sargassum binderi in oleic acid vesicular system. This resulted in nanosized-formulation (PNV) which elicited a spectrum of 
antibacterial effects in vitro, thereby revealing promising potential as an effective therapeutic agent against human pathogenic bacteria.

\section{MATERIAL AND METHODS}

\section{Chemicals and Reagents}

All organic solvents and oleic acid used in this study were purchased from Sigma Aldrich, USA. Bacteriological media were products of Scharlau (Afaq Sada Trading, Riyadh, KSA), and Somatco (Jeddah, KSA).

\section{Seaweed collection and identification}

The seaweeds were collected at predetermined time intervals from January to June 2017 from Al Murjan beach, Jazan, Kingdom of Saudi Arabia. The seaweeds were collected during low tide from intertidal zones of the sea near the shore, and $1 \mathrm{~km}$ inside the sea (Figure 1). The seaweed specimens were collected from Al Murjan beach of Red sea, Jazan, KSA and identified in the Herbarium of Jazan University (JAZUH). A voucher specimen was also deposited at the Herbarium for further reference. The collected seaweed was identified as Sargassum binderi Sonder ex J.Agardh and the reference number was 1210(JAZUH).

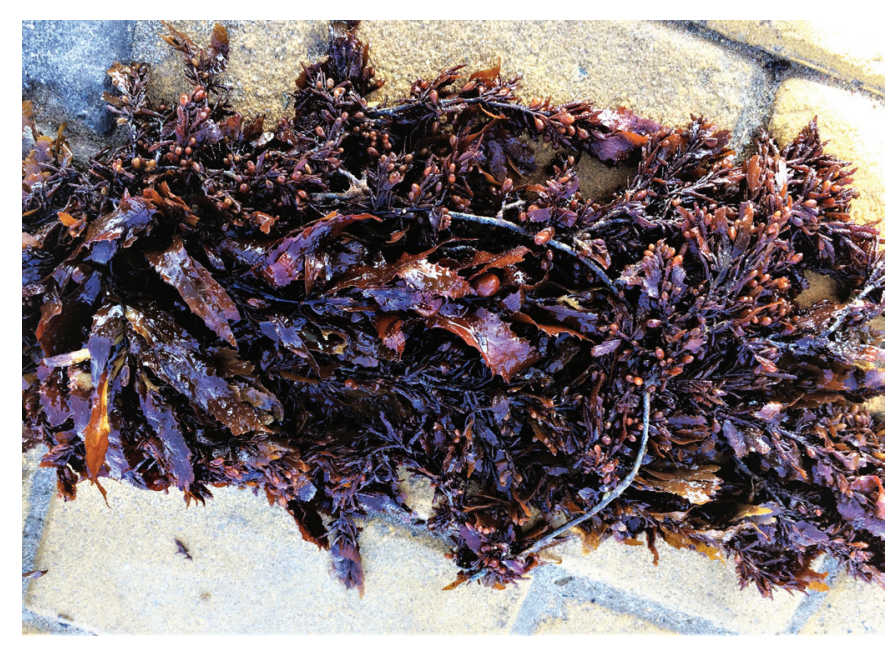

FIGURE 1 - Sargassum binderi Sonder ex J. Agardh.

\section{Seaweed processing}

The seaweeds were washed thoroughly with fresh water in order to remove unwanted materials. After cleaning, the samples were initially air-dried and further dried in the shade at room temperature for a period of two weeks. The dried samples were cut into small pieces and powdered using a grinder. The powdered sample was kept at room temperature in an air-tight container.

\section{Seaweed extraction process}

Maceration, decoction and hot continuous percolation were employed in this study to isolate various phyto-constituents from the powdered sample.

\section{Maceration method}

Weighed powder $(25 \mathrm{~g})$ was soaked in $500 \mathrm{~mL}$ of methanol for a period of one week and then filtered through Whatman filter paper No. 1. The extract was dried at room temperature, and then subjected to phytochemical analysis prior to use for antibacterial studies.

\section{Decoction method}

Powdered sample (25 g) was boiled in $250 \mathrm{~mL}$ of millipore water and filtered using conventional tea filter. The filtrate was subjected to phytochemical analysis before its use for antibacterial assays.

\section{Hot continuous percolation using Soxhlet extraction}

The extraction was done as established by Sivakumar et al. (2013). In this method, $200 \mathrm{~g}$ of finely-powdered Sargassum binderi was packed in Soxhlet apparatus, and $500 \mathrm{~mL}$ of petroleum ether was put in the round bottom flask. The total assembly was placed on a heating mantle and the solvent was heated continuously at $60{ }^{\circ} \mathrm{C}$ for 4 $\mathrm{h}$. The extracts were transferred separately into open glass beakers, and the solvent was allowed to evaporate through air-drying. The dried samples were scrapped off, pooled, weighed and kept in an air-tight container. The percentage yield of the extract was then calculated, prior to antibacterial studies.

\section{Phytochemical analysis}

The three extracts were subjected to quantitative phytochemical analysis for alkaloids, carbohydrates, proteins, amino acids, tannins, steroids and saponins. Phytochemical screening was performed according to procedures reported earlier (Sivakumar et al., 2013, 2008; Sarfaraj Hussein et al., 2012).

\section{Bacterial strains and standardization}

Twenty-four-hour (24-h) cultures of Staphylococcus aureus, Streptococcus pyogenes, Bacillus subtilis, Klebsiella pneumoniae, Escherichia coli and Pseudomonas aeruginosa were utilized in this study. The cultures were 
diluted in gradient concentration from $10^{-1}$ to $10^{-9}$ in sterilized nutrient broth. The potential viabilities of the bacterial organisms were determined by assessing the colony forming unit in $1 \mathrm{~mL}(\mathrm{CFU} / \mathrm{mL})$.

\section{Minimum inhibitory concentration (MIC) study}

The minimum inhibitory concentrations (MICs) were determined as established by Sivakumar et al. (2013). The formulation of PNV was based on the MICs of the seaweed extract.

\section{Preparation of PNVs}

The PNVs were prepared using a simple film hydration method modified from that previously reported by Foziyah et al. (2010) and Verma et al. (2013). In essence, 2 $\mathrm{mL}$ of test sample at a concentration of $200 \mu \mathrm{g}$ extract/100 $\mu \mathrm{L}$ was mixed with $2 \mathrm{~mL}$ of methanol and then oleic acid in $1: 1$ volume ratio. The mixture was sonicated for $5 \mathrm{~min}$ at $40 \%$ amplification, and thereafter it was placed on a rotary shaker at $200 \mathrm{rpm}$ for $20 \mathrm{~min}$. The same procedure was repeated twice to develop the PNVs. The nanovesicles were further developed by heating in a heating mantle for about $60 \mathrm{~min}$ at $50{ }^{\circ} \mathrm{C}$ to form a thin film. Finally, the film was eluted in methanol and used for antibacterial studies.

\section{Physical characterization}

Methanol-eluted PNVs was diluted in Millipore water at a volume ratio of $1: 1$ and the physical characteristics such as zeta potential, size (z- d.nm) and pdi were determined using Zeta Sizer Nano ZS (Malvern Instruments, UK). The standard operating procedure was followed as per the procedures outlined in the Zeta Nano Sizer user manual.

\section{Scanning Electron Microscopy (SEM) and Energy Dispersive Spectroscopy (EDAX) analysis}

The morphological features and the particle size in diameter were studied using high resolution SEM. A drop of PNVs was placed on a clean glass slide $3 \mathrm{~cm} \times 3 \mathrm{~cm}$ in size and air-dried in a laminar hood. Then, the sample was coated with gold and the images were observed at various magnifications. Energy Dispersive Spectroscopy (EDAX) is a useful technique for determining the elemental compositions of samples. The EDAX spectrum was obtained at an acceleration voltage of $5 \mathrm{keV}$ and collected at 31s. The SEM and EDAX were obtained using FEI Quanta 200 F (FEI Company, USA).

\section{In vitro antibacterial screening}

The in vitro antibacterial screening was performed as established by Sivakumar et al. (2013, 2008). A known concentration of Muller Hinton agar media was prepared and plated under aseptic conditions. The agar well diffusion technique was used for the antibacterial susceptibility test of the crude extracts, DMSO (solvent control) and oleic acid (adjuvant control), whereas agar disc diffusion method was applied in determining the antibacterial susceptibility test of the standard ciprofloxacin disc $(5 \mu \mathrm{g} / \mathrm{disc})$.

\section{STATISTICAL ANALYSIS}

All the experiments were performed six times $(n=6)$ and the data were subjected to one-way analysis of variance (ANOVA). The levels of statistical significance were $p<0.001$ (very highly significant), $p<0.01$ (highly significant) and $p<0.05$ (significant). All statistical analyses were done using the Graph Pad Instat software system. The test values were compared with the standard drug values using Dunnet's post hoc test).

\section{RESULTS AND DISCUSSION}

The discovery of antibiotics and antibacterial agents is significant for combating bacterial infections in the modern therapeutic system of medicine. Bacterial resistance is a prominent event that occurs due to various mechanisms such as efflux pumps and exocytosis. Drug resistance poses a serious challenge to physicians in the treatment of diabetic foot ulcers due to poor delivery of drugs to the tissues. Therefore, in order to combat this problem, diverse efforts have been devoted to the development of newer drugs, especially antibiotics due to the frequent occurrence of resistance as a result of drug misuse and irrational drug therapy (Sivakumar et al., 2016). Natural products are unique resources for bioactive substances, having high molecular diversity and promising pharmacological actions. In recent years, drug development has focused on marine natural products because marine sources are a remarkable reservoir of many novel pharmaceutically-active compounds (Namvar, Baharara, Mahdi, 2014; Sivakumar et al., 2013; Sarfaraj Hussein et al., 2012). The brown alga Sargassum binderi Sonder ex J. Agardh was collected in intertidal zones, a very rare kind of seaweed habitat in the Red Sea, Jazan, KSA. The seaweed (Figure 1) is largely present during the months of December to February. The seaweeds collected from inside the sea were fresher than the samples 
collected near the sea shore. The petroleum ether extract of seaweed using hot continuous percolation method showed various phytoconstituents such as carbohydrates, tannins, steroids and saponins (Table I). However, tannins and steroids were the predominant phyto-constituents seen in this study. The method of extraction influences the nature of phyto-constituents extracted. In this study, it is very obvious that tannins and steroids were isolated only by Soxhlet extraction. In 2016, Lim et al. (2016) reported that fucoidan, a sulphated polysaccharide from Malaysian brown seaweed, was isolated from Sargassum binderi Sonder ex J. Agardh, while Irwandi Jaswir et al. (2012) isolated the lipid component fucoxanthin from Sargassum binderi. Although a few researchers have demonstrated some bioactive properties of Sargassum binderi, the antibacterial properties have not been studied yet. Interestingly, the in vitro antibacterial analysis of phytoextracts in the present study revealed a good spectrum of activity against the screened organisms which may be due to the presence of tannins. Studies by Abdul
Rahim et al. (2012) demonstrated the anti-bacterial effect of crude acetone and ethanol extracts of brown seaweed Sargassum boveanum against E. coli-induced diarrhea in children.

The nanovesicles were successfully formulated and their physicochemical characterization was carried out. The properties of the PNVs are shown in Table II. It is obvious that uniform nanovesicles were obtained, with zeta potential of $-27.4 \pm 4 \mathrm{mV}$; pdi of 0.321 , and the average size in diameter was about $112.7 \mathrm{z}$-d.nm (Figures $2 \mathrm{~A} \& 2 \mathrm{~B}$ ). The potential viability of bacterial culture was assessed by preparing $24 \mathrm{~h}$ culture and standardization by serial dilution technique using nutrient broth. The concentration of microbial culture varied with individual bacterial organisms (Table III). The MICs of the extracts for the screened organisms were between $100-200 \mu \mathrm{g} / 100 \mu \mathrm{L}$ of the test sample, depending on the organism. Therefore, the extract concentration of $200 \mu \mathrm{g} / 100 \mu \mathrm{L}$ was selected for use in the formulation of the PNVs. The zeta potential, which is expressed in

TABLE I - Qualitative phytochemical analysis of Sargassum binderi by using different extraction methods

\begin{tabular}{lccc}
\hline Name of the test & $\begin{array}{c}\text { Hot continuous percolation by Soxhlet } \\
\text { apparatus using petroleum ether }\end{array}$ & Decoction & Maceration \\
\hline Percentage of yield & 1 & 0.25 & 0.4 \\
Carbohydrates & + & + & + \\
Reducing sugar & - & - & - \\
Ketohexose sugar & - & - & - \\
Aldohexoses sugar & + & + & + \\
Proteins & - & - & - \\
Amino acids & - & - & - \\
Steroids & $+($ Predominant $)$ & - & - \\
Alkaloids & - & - & - \\
Tannins & $+($ Predominant $)$ & - & + \\
Saponins & + & + & \\
\hline
\end{tabular}

* (+) yes; (-) no

TABLE II - Characterization of Phyto nanovesicles (PNV)

\begin{tabular}{|c|c|c|c|c|c|c|c|c|c|c|c|}
\hline \multicolumn{3}{|c|}{$\begin{array}{c}\text { Physical characterization by } \\
\text { Zeta sizer analysis }\end{array}$} & \multicolumn{3}{|c|}{$\begin{array}{c}\text { Morphological appearance } \\
\text { by SEM analysis }\end{array}$} & \multicolumn{6}{|c|}{ Elements (Wt\%) by EDAX analysis } \\
\hline $\begin{array}{c}\text { Zeta } \\
\text { potential } \\
(\mathrm{mV}) \\
\end{array}$ & $\begin{array}{l}\text { Size } \\
(\text { d.nm })\end{array}$ & pdi & $\begin{array}{l}\text { Surface } \\
\text { character }\end{array}$ & Shape & $\begin{array}{c}\text { Size in } \\
\text { diameter } \\
(\mathrm{nm} / \mu \mathrm{m})\end{array}$ & C & $\mathbf{O}$ & $\mathbf{N a}$ & $\mathbf{K}$ & Al & Ti \\
\hline$-27.4 \pm 4$ & 100 & 0.321 & Smooth & Spherical & $\begin{array}{l}20-23 \\
2-10^{*}\end{array}$ & 42.51 & 20.35 & 5.39 & 1.84 & 1.75 & 1.82 \\
\hline
\end{tabular}

* Size in $\mu \mathrm{m}$ level. Abbreviations: $\mathrm{mV}$ - millivolt; d.nm - Diameter in nanometer; pdi - polydispersity index; nm- nanometer, $\mu \mathrm{m}$ - micrometer 
Results

Zeta Potential (mV): -27.4

Zeta Deviation (mV): 4.84

Conductivity (ms/cm): 0.822

Result quality : Good
$\mathbf{A}$

$\begin{array}{llll} & \text { Mean }(\mathrm{mV}) & \text { Area }(\%) & \text { StDev }(\mathrm{mV}) \\ \text { Peak 1: } & -27.4 & 100.0 & 4.84 \\ \text { Peak 2: } & 0.00 & 0.0 & 0.00 \\ \text { Peak 3: } & 0.00 & 0.0 & 0.00\end{array}$

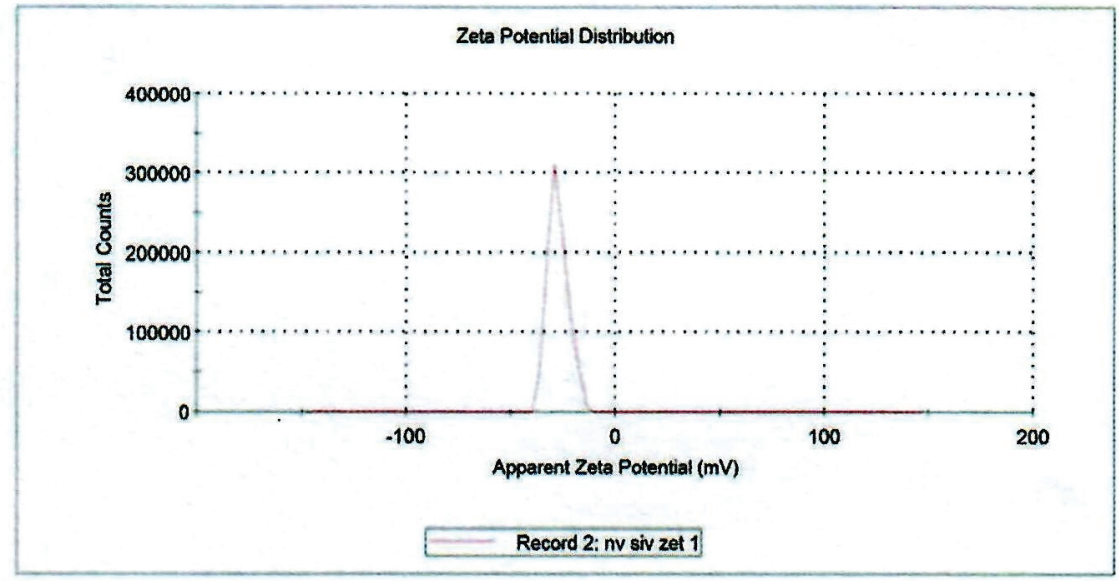

Results

B

$\begin{array}{rlllll} & & & \text { Size (d.nm): } & \text { \% Intensity: } & \text { St Dev (d.n... } \\ \text { Z-Average (d.nm): } & 112.7 & \text { Peak 1: } & 115.7 & 81.2 & 60.95 \\ \text { Pdl: } & 0.321 & \text { Peak 2: } & 860.9 & 17.7 & 513.4 \\ \text { Intercept: } & 0.924 & \text { Peak 3: } & 4429 & 1.1 & 868.5 \\ \text { Result quality : } & \text { Good } & & & & \end{array}$

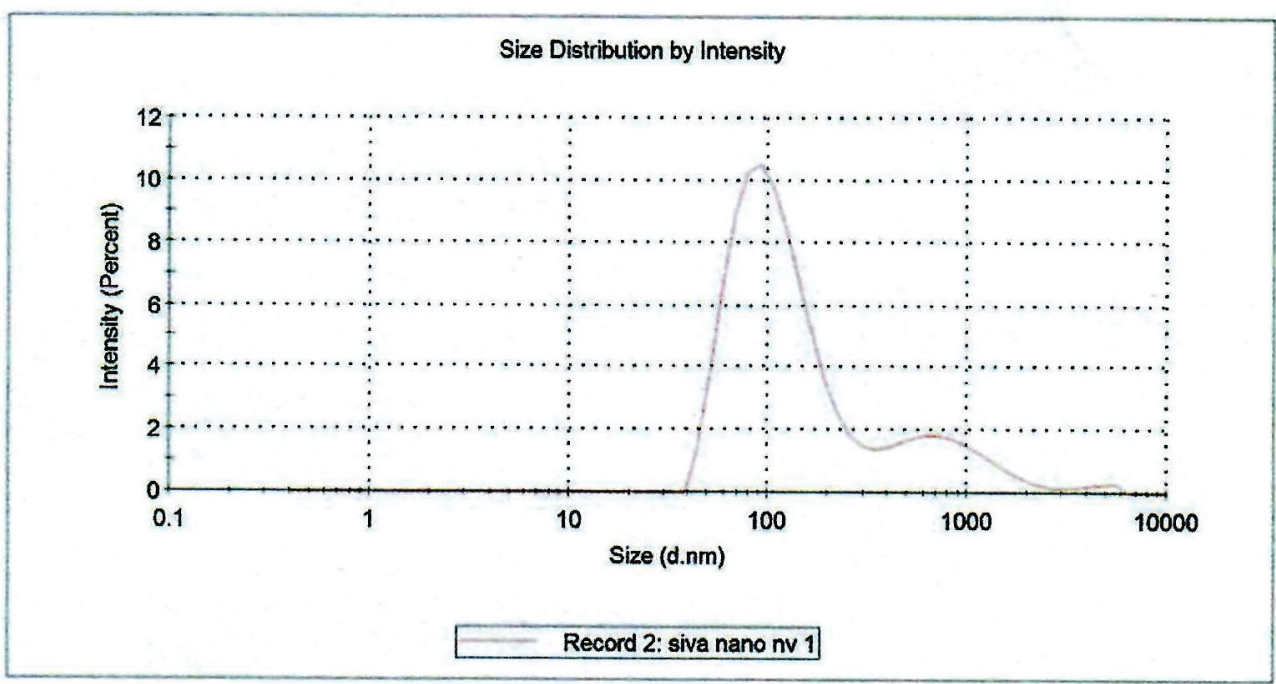

FIGURE 2 - Zeta potential analysis of phyto nanovesicles (PNV). (A) Zeta potential of PNV prepared by using petroleum ether extract of Sargassum binderi with oleic acid; (B) Zeta size and pdi of PNV prepared by using petroleum ether extract of Sargassum binderi with oleic acid.

millivolts $(\mathrm{mV})$ is an important factor for assessing the penetrating capability of PNVs into bacterial cells. The zeta potential analysis of the PNVs and bacterial organisms at specific concentrations are presented Tables II and III, respectively.
The result of SEM analysis of PNV at 100,000 magnification are shown in Figure $3 \mathrm{~A}$. The PNV morphology revealed even and spherical shapes within the sizes $20-23 \mathrm{~nm}$. However, the size of very a few particles was up to $10 \mu \mathrm{m}$. The SEM analysis of a single 
PNV at 15,000 $\times$ magnification revealed a smooth spherical morphology of size $2.69 \mu \mathrm{m}$ but few vesicles were about $9.42 \mu \mathrm{m}$ in size (Figures 3B \& 3C). Figure 3D shows the energy dispersive spectrum of the formulated PNVs, suggesting the presence of $\mathrm{C}, \mathrm{O}, \mathrm{Na}, \mathrm{Al}, \mathrm{Cl}, \mathrm{K}$ and Ti. The predominant peak near $2 \mathrm{kev}$ indicates the presence of $\mathrm{Si}$, most likely from the glass slide used to mount the nanovesicles. The peak at $2 \mathrm{kev}$ indicates the presence of Au (gold) which was used to coat the samples for conductivity. Table IV depicts the antibacterial effects of petroleum ether extract of Sargassum binderi and their nanovesicles against human pathogenic bacteria. The results demonstrated that the crude petroleum ether phytoextract depicted good activity against human pathogenic bacteria. However, the extract was formulated into PNV using oleic acid with the aim of improving its activity. The results showed that the nanovesicle formulation elicited higher antibacterial effect than the crude extract. Since oleic acid was used to formulate the PNVs, and methanol was used as eluting solvent while
DMSO was used as a solvent for antibacterial studies, these solvents were also screened individually for antibacterial effects. However, no significant antibacterial effect was seen against the screened bacteria. From the results, it is very obvious that the PNVs possess a promising spectrum of in vitro antibacterial activity comparable to that of standard ciprofloxacin disc. An earlier report showed that oleic acid vesicles can be used as a delivery system for topical applications containing antifungal agents (Verma et al., 2010). The spectrum of antibacterial effects of particles is based on characteristics such as surface potential and shape. Obviously, smaller particles will show better antibacterial activity irrespective of surface charge due to easy penetration through the endocytosis process (Lucia et al., 2017; Abbas et al., 2015). In this study the nanovesicles elicited better antibacterial action when compared to the crude extract. The increase in spectrum of activity was probably due to the easy penetration of the PNVs as was reflected in zeta analysis and SEM analysis. In addition, the formulation was more

TABLE III - Zeta potential of screened bacteria at specified concentration

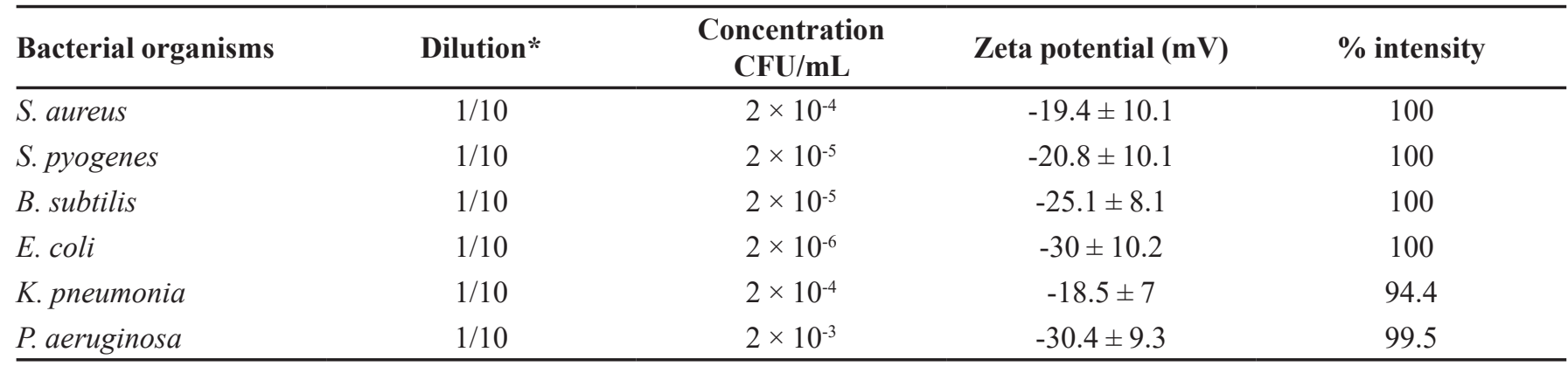

* Dilution of 24 hours culture in Millipore water

TABLE IV - A Comparative antibacterial study of PNV

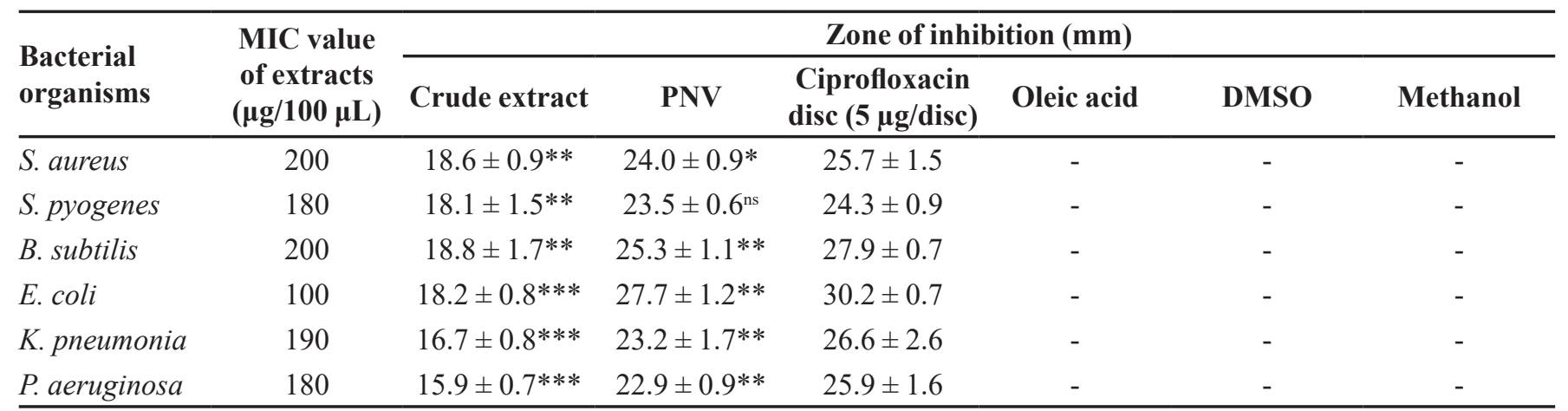

(-) No activity. Each value is the mean of $n=6$ batches with standard deviation by performing Dunnett multiple comparison test (Sample VS Standard), (post hoc test). ${ }^{* * *} P<0.001$ (99\% confidence interval) is very extremely significant when compared to standard ciprofloxacin disc; ${ }^{* *} P<0.01$ (99\% confidence interval) is highly significant when compared to standard ciprofloxacin disc; ${ }^{*} P<0.05$ (95\% confidence interval) is significant when compared to standard ciprofloxacin disc; ns - non-significant when compared to standard ciprofloxacin disc. Abbreviations: $\mathrm{mm}$ - millimeter; $\mu \mathrm{g}$ - microgram; $\mathrm{mL}-\mathrm{milliliter}$. 


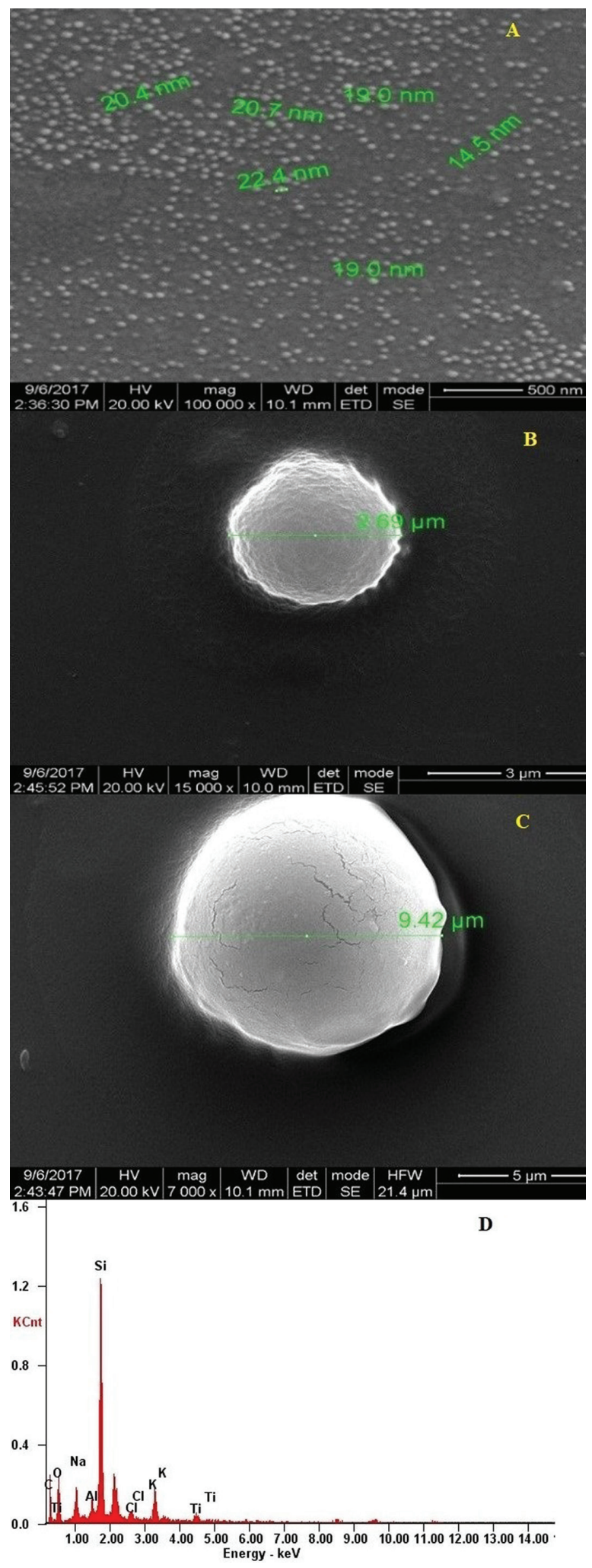

FIGURE 3 - SEM and EDAX analysis of phyto nanovesicles (PNV). (A) Scanning electron micrograph of PNV at 100,000 $\times$ magnification; (B) Scanning electron micrograph of PNV at $15,000 \times$ magnification (size in $\mu \mathrm{m}) ;(\mathrm{C})$ Scanning electron micrograph of PNV at $7000 \times$ magnification (size in $\mu \mathrm{m})$; (D) EDAX analysis of PNV. successful than the crude extract because of ideal physical properties such as uniformity in distribution. Studies by Abbas et al. (2015) on the effect of particle charge on the antibacterial properties demonstrated that positivelycharged silver nanoparticles were greatly attracted to the negative charge of bacterial cells. Furthermore, the study also reported that the repulsive effect of negative silver nanoparticles resulted in electrostatic barrier which led to limited effectiveness. In contrast to these reports, the present study demonstrated that the PNVs exhibited a spectrum of antibacterial effects against the screened organisms. The PNVs exhibited zeta potential of $-27.4 \pm 4 \mathrm{mV}$, indicating that the vesicular system was highly stable. Interestingly, the zeta potential analysis of the screened bacteria exhibited varied zeta potential which were individually unique at the screened concentration. Bacterial surface charge is an electro-chemical property of the cell surface arising from the presence of various functional groups that vary between Gram-positive and Gram-negative bacteria (Suman et al., 2015). These functional groups are teichoic acid and peptidoglycan of Gram-positive bacteria (Yongsuk, Brown, 2006); and lipopolysaccharides and phospholipids of Gram-negative bacteria. Although the surface charge is anionic, the vesicles could easily penetrate the bacterial cell through endocytosis, and exhibit the action seen in vitro screening. Thus, in this study the size of the PNV ranged from $10-$ $20 \mathrm{~nm}$ which was reflected in the SEM analysis and the wider spectrum of antibacterial effect. The SEM study demonstrated that most of the particles were much smaller in size (14 - $23 \mathrm{~nm})$. The surface was partially smooth and spherical in shape. However, the study shows that the size of few vesicles was about $2-10 \mu \mathrm{m}$. This was reflected in zeta size analysis, where it was observed that a few particles had a higher size range. However, the zeta d.nm of majority of particles was 112.5 d.nm. The PNVs manifested a promising spectrum of anti-bacterial effects against selected Gram-positive and Gram-negative bacteria. Although the surface charge is an important factor for targeting the cell, size is also another important determinant of the penetration of particles into the targeted cell. Thus, charge distribution is not the only factor that determines penetration into the cell. Furthermore, studies have shown that negatively-charged particles are safer than positively charged nanoparticles which may cause damage to the cell wall/cell membrane (Yang, Lee, Too, 2007). These studies have shown that stable vesicular system was successfully developed by entrapping petroleum ether extract of Sargassum binderi. The energy dispersive X-ray spectrometry (EDAX) analysis revealed that the PNVs contained $42.51 \%$ carbon and $20.35 \%$ oxygen, as well as 
the presence of other trace elements such as $\mathrm{Na}, \mathrm{K}, \mathrm{Cl}$ and Ti. In a study by Apaydn et al. (2010), the metal contents of seaweed Ulva lactuca were determined using energydispersive X-ray fluorescence spectrometry (EDXRF). The PNV exhibited a broad spectrum of activity against all the organisms screened and the spectrum of activity was comparable to that of standard ciprofloxacin.

\section{CONCLUSION}

The seaweed Sargassum binderi from the Red Sea, Jazan, KSA is a very rare species and has not yet been studied properly for its biological activity. The results obtained in this study indicate that anti-bacterial principles can be isolated from Sargassum binderi. However, the spectrum of activity was increased by PNV formulation which can be considered as a highly promising novel technique for delivering bioactive substances inside the cell. The results reveal promising potential of PNV as an effective therapeutic agent against human pathogenic bacteria, and have opened a new gateway by developing a successful formulation technique for delivery of antibacterial agents.

\section{ACKNOWLEDGEMENT}

The authors are acknowledging the Deanship of Scientific Research, Jazan University for funding the project, Reference No. 37/7/000102. The investigators also thank Ms. Kalpana, Project Associate, HR - SEM, SAIF, IIT, Chennai, India for performing SEM \& EDAX analysis of PNV.

\section{CONFLICT OF INTEREST}

Authors declare that there is no conflict of interest is associated with this study.

\section{REFERENCES}

Abbas A, Yasamin G, Ahmad G, Bahram H, Samira D, Mohammadreza N, et al. The effect of charge at the surface of silver nanoparticles on antimicrobial activity against Grampositive and Gram-negative bacteria: A Preliminary Study. J Nanomater. 2015;2015:1-8.

Abdul RK, Rashida Q. Antibacterial activities of brown seaweed Sargassum boveanum (J.AG.) against diarrhea along the coast of Karachi, Pakistan. J Environ Res Develop. 2012;6(3A):753-757.
Apaydin G, Ayliker A, Cengiz E, Saydam M, Kup N, Tirasoglu E. Analysis of metal contents of seaweed (Ulva lactuca) from Istanbul, Turkey by EDXRF. Turk J Fish Aquat Sci. 2010;10(2):215-220.

Collis CM, Hall RM. Expression of antibiotic resistance genes in the integrated cassettes of integrons. Antimicrob Agents Chemother. 1995;39(1):155-162.

Cosgrove SE. The relationship between antimicrobial resistance and patient outcomes: mortality, length of hospital stay, and health care costs. Clin Infect Dis. 2006;42(Suppl 2):S82-89.

Foziyah Z, Bhuvaneshwar V, Amit KG, Basant PV. Development and characterization of oleic acid vesicles for the topical delivery of fluconazole. Drug Deliv. 2010;17(4):238-248.

Irwandi J, Dedi N, Hamazh MS, Kazuo M. Fucoxanthin extractions of brown seaweeds and analysis of their lipid fraction in methanol. Food Sci Technol Res. 2012;18(2):251- 257.

Lim SJ, Wan Aida WM, Maskat MY, Latip J, Badri KH, Hassan $\mathrm{O}$, et al. Characterisation of fucoidan extracted from Malaysian Sargassum binderi. Food Chem. 2016;15(209):267-273.

Lucia S, Elisabetta G, Veronica C, Giulia A, Svetlana A, Fabio C, et al. Negatively charged silver nanoparticles with potent antibacterial activity and reduced toxicity for pharmaceutical preparations. Int J Nanomedicine. 2017;12:2517-2530.

Maria JP, Elena F, Herminia D. Anti-microbial action of compounds from marine seaweed. Mar Drugs. 2016;14(52):138.

Namvar F, Baharara J, Mahdi AA. Antioxidant and anticancer activities of selected Persian Gulf Algae. Indian J Clin Biochem. 2014;29(1):13-20.

Pelgrift RY, Friedman AJ. Nanotechnology as a therapeutic tool to combat microbial resistance. Adv Drug Deliv Rev. 2013;65(13-14):1803-1815.

Riley MA, Robinson SM, Roy CM, Dennis M, Liu V, Dorit RL. Resistance is futile: The bacteriocin model for addressing the antibiotic resistance challenge. Biochem Soc Trans. 2012;40(6):1438-1442.

Sarfaraj Hussein Md, Sheeba F, Saba A, Sajid KM. Marine natural products: A lead for anti-cancer. Indian J Mar Sci. 2012;41(1):27-39. 
Sivakumar SM, Raviraj M, Lavakumar V, Anbu J, Shanmugarajan TS, Muregasan R, et al. Isolation and screening of bioactive principles from Hypnea musciformis (Wulfen) Lamouroux against cancer. Seaweed Res Utilin. 2008;30(1-2):119-124.

Sivakumar SM, Safhi MM. Isolation and screening of bioactive principle from Chaetomorpha antennina against certain bacterial strains. Saudi Pharm J. 2013;21(1):119-121.

Sivakumar SM, Safhi MM, Aamena J, Foziyah Z, Farah I, Bagul US, et al. Therapeutic potential of chitosan nanoparticles as antibiotic delivery system: challenges to treat multiple drug resistance. Asian J Pharm. 2016;10(2):S61-66.

Suman H, Kirendra KY, Ratul S, Sudipta M, Pritam S, Saubhik $\mathrm{H}$, et al. Alteration of Zeta potential and membrane permeability in bacteria: a study with cationic agents. Springer Plus. 2015;4(672):1-14.
Verma S, Bhardwaj A, Mohit V, Pawan B, Nishant G, Kumar L. Oleic acid vesicles: a new approach for topical delivery of antifungal agent. Artif Cells Nanomed Biotechnol. 2014;42(2):95-101.

Yang J, Lee JY, Too HP. A general phase transfer protocol for synthesizing alkaline - stabilized nanoparticles of noble metals. Anal Chim Acta. 2007;588(1):34-41.

Yongsuk H, Brown DG. Cell surface acid-base properties of Escherichia coli and Bacillus brevis and variation as a function of growth phase, nitrogen source and C:N ratio. Colloids Surf B Biointerfaces. 2006;50(2):112-119.

Received for publication on $13^{\text {th }}$ December 2017 Accepted for publication on $28^{\text {th }}$ September 2018 